\title{
Fundamental properties of extended Horadam numbers
}

\author{
Gülsüm Yeliz Şentürk ${ }^{1}$, Nurten Gürses ${ }^{2}$ and Salim Yüce ${ }^{3}$ \\ ${ }^{1}$ Faculty of Engineering and Architecture, Istanbul Gelisim University, \\ Department of Computer Engineering, 34310, Istanbul, Turkey \\ e-mail: gysenturk@gelisim.edu.tr \\ ${ }^{2}$ Faculty of Arts and Sciences, Yildiz Technical University, \\ Department of Mathematics, 34220, Istanbul, Turkey \\ e-mail: nbayrakeyildiz.edu.tr \\ ${ }^{3}$ Faculty of Arts and Sciences, Yildiz Technical University, \\ Department of Mathematics, 34220, Istanbul, Turkey \\ e-mail: sayucedyildiz.edu.tr
}

\begin{abstract}
In this paper, the extended Horadam numbers are introduced by using dual-generalized complex, hyperbolic-generalized complex and complex-generalized complex numbers. Then, generating function, Binet's formula, D'Ocagne's, Catalan's and Cassini's identities are given. Moreover, special matrix representations of the extended Horadam numbers are investigated. In conclusion, the results and classification of the special cases are introduced.
\end{abstract}

Keywords: Horadam number, Dual-generalized complex number, Hyperbolic-generalized complex number, Binet's formula, D’Ocagne's identity, Catalan's identity, Cassini's identity.

2020 Mathematics Subject Classification: 11B37, 11B39, 11K31.

\section{Introduction}

Recurrence sequences have been a central part of number theory for many years. These sequences are applied to many areas of mathematics and computer science. Linear recurrence sequences have arisen in computer graphics, approximation theory, cryptography and time series analysis. 
Horadam sequence $W_{n}(a, b ; p, q)$, so named after the studies of A. F. Horadam begun in the 1960s, is a particular type of linear recurrence sequences. It gives rise to some well known sequences such that Fibonacci, generalized Fibonacci, Lucas, Pell, Pell-Lucas, Jacobsthal, Jacobsthal-Lucas, Tagiuri, Fermat, Fermat-Lucas, and so on. These sequences have applications in science and nature. For example, the applications of the golden ratio (the limit of the ratio of two consecutive Fibonacci numbers) appear in many research areas, particularly in physics, engineering, architecture, nature and art.

The Horadam sequence has ensured wide mathematical contribution to the academic community, thus many publications either directly on, or relating to, have appeared in the literature, [16-20, 27]. In [23] more general identities involving the terms Horadam sequence and in [3] some generalizations of Horadam's numbers are examined. Also, Horadam numbers are studied in $[34,37]$.

On the other side, the set of generalized complex numbers is defined as, [15,21]:

$$
\mathbb{C}_{\mathfrak{p}}:=\left\{z=a_{1}+a_{2} J \mid a_{1}, a_{2} \in \mathbb{R}, J^{2}=\mathfrak{p} \in \mathbb{R}, J \notin \mathbb{R}\right\}
$$

The vector space $\mathbb{C}_{\mathfrak{p}}$ (over $\mathbb{R}$ ) is analogue to complex numbers for $\mathfrak{p}=-1$, hyperbolic numbers (double, split complex, perplex) for $\mathfrak{p}=1$, and dual numbers for $\mathfrak{p}=0$ (see details in [29, $31,32,35,36]$ ). Rebuilding new numbers with the coefficients of above number systems is an attractive area for researchers and that enables to construct different types of number systems (see in $[1,5,6,8-10,21,25,26]$ ).

In [12], as a new approach, dual-generalized complex $(\mathcal{D G C})$, hyperbolic-generalized complex $(\mathcal{H G C})$ and complex-generalized complex $(\mathcal{C G C})$ numbers are introduced by using the Cayley-Dickson doubling procedure. For the special real values $\mathfrak{p}=-1, \mathfrak{p}=0$ and $\mathfrak{p}=1$, dual-complex, hyper-dual, dual-hyperbolic, hyperbolic-complex, bihyperbolic and bicomplex numbers are obtained from $\mathcal{D G C}, \mathcal{H G C}$ and $\mathcal{C G C}$ numbers (see detailed classification in [12]). As we move from number systems to elements of Horadam sequences, some papers can be examined in literature, $[2,4,7,11,13,14,24,28,33]$.

In this present study, firstly, algebraic properties and linear recurrence relations for $\mathcal{D G C}$ Horadam numbers $\tilde{\mathcal{W}}_{n}$ are introduced. Furthermore, generating functions, Binet's formula, D'Ocagne's, Catalan's and Cassini's identities are computed for $\mathfrak{p} \in \mathbb{R}$. With the same approach, the fundamental formulas for Horadam numbers with $\mathcal{H G C}$ and $\mathcal{C G C}$ coefficients are stated. A series of matrix representations of these Horadam numbers is examined. The multiplication of Horadam numbers is also expressed as their matrix representations. The main importance in carrying out this construction is that dual-complex, hyper-dual, dual-hyperbolic, hyperboliccomplex, bihyperbolic and bicomplex Horadam numbers can be figured out by the readers for the specific values $\mathfrak{p} \in\{-1,0,1\}$.

\section{Basic concepts}

In this part of the paper, we present some needed basic results related to Horadam sequence and $\mathcal{D G C}, \mathcal{H G C}$ and $\mathcal{C G C}$ numbers. 


\subsection{Horadam numbers}

Definition 2.1. For $a, b, p, q \in \mathbb{Z}$, the generalized sequence of $W_{n}(a, b ; p, q)$, briefly $W_{n}$, satisfies the following second order recurrence relation

$$
W_{n}(a, b ; p, q)=p W_{n-1}-q W_{n-2}, \quad(n \geq 2),
$$

where initial conditions $W_{0}=a, W_{1}=b$. In honor of Horadam, this general sequence is called a Horadam sequence ${ }^{1}$, [18-20].

The characteristic equation related to the recurrence relation (1) is $x^{2}-p x+q=0$ with characteristic roots $\alpha$ and $\beta$ that satisfy:

$$
\alpha+\beta=p, \quad \alpha \beta=q, \quad \alpha-\beta=\sqrt{p^{2}-4 q} .
$$

Then, the Binet's formula of Horadam numbers is as follows:

$$
W_{n}=A \alpha^{n}+B \beta^{n},
$$

where $A=\frac{b-a \beta}{\alpha-\beta}$ and $B=\frac{a \alpha-b}{\alpha-\beta},[18]$.

Corollary 2.1. The following linear recurrence relations hold:

- $W_{n+2}=\left(p^{2}-q\right) W_{n}-p q W_{n-1},[19]$

- $p W_{n+2}=\left(p^{2}-q\right) W_{n+1}-q^{2} W_{n-1}$, [19]

- $\left(p^{2}-2 q\right) W_{n+2}=q^{2} W_{n}+W_{n+4},[30]$,

- $\left(p^{4}-3 p^{2} q+q^{2}\right) W_{n+2}=\left(p^{2} q^{2}-q^{3}\right) W_{n}+p W_{n+5}$, [30],

- $\left(p^{4}+3 q^{2}-4 p^{2} q\right) W_{n+2}=\left(p^{2} q^{2}-2 q^{3}\right) W_{n}+W_{n+6}$, [30],

- $\left(p^{4}-5 p^{4} q+6 p^{2} q^{2}-q^{3}\right) W_{n+2}=\left(p^{4} q^{2}-3 p^{2} q^{3}+q^{4}\right) W_{n}+p W_{n+7}$, [30],

- $W_{k+n}=\left(\alpha^{n}+\beta^{n}\right) W_{k}-q^{n} W_{k-n},[34]$.

Furthermore, the generating function of Horadam numbers is

$$
g(x)=\sum_{n=0}^{\infty} W_{n} x^{n}=\frac{W_{0}+\left(W_{1}-p W_{0}\right) x}{1-p x+q x^{2}},
$$

where $W_{0}, W_{1}$ are initial Horadam numbers, [18].

${ }^{1}$ Special Horadam numbers:

- $W_{n}(0,1 ; 1,-1)=F_{n}$ : Fibonacci

- $W_{n}(2,1 ; 1,-1)=L_{n}$ : Lucas

- $W_{n}(0,1 ; 2,-1)=P_{n}$ : Pell

- $W_{n}(1,1 ; 2,-1)=M P_{n}$ : Modified Pell

- $W_{n}(2,1 ; 1,-2)=j_{n}$ : Jacobsthal-Lucas

- $W_{n}(1,3 ; 3,-2)=T_{n}$ : Fermat
- $W_{n}(0,1 ; p, q)=U_{n}$ : Generalized Fibonacci

- $W_{n}(2, p ; p, q)=V_{n}$ : Generalized Lucas

- $W_{n}(2,2 ; 2,-1)=Q_{n}$ : Pell-Lucas

- $W_{n}(0,1 ; 1,-2)=J_{n}$ : Jacobsthal

- $W_{n}(0,1 ; 3,2)=M_{n}$ : Mersenne 


\section{$2.2 \mathcal{D G C}, \mathcal{H G C}$ and $\mathcal{C G C}$ numbers}

The set of $\mathcal{D G C}$ numbers is introduced as: $\mathbb{D C}_{\mathfrak{p}}:=\left\{w=z_{1}+z_{2} \varepsilon \mid z_{1}, z_{2} \in \mathbb{C}_{\mathfrak{p}}, \varepsilon^{2}=0, \varepsilon \neq 0, \varepsilon \notin \mathbb{R}\right\}$. For $w_{1}=z_{11}+z_{12} \varepsilon, w_{2}=z_{21}+z_{22} \varepsilon \in \mathbb{D C}_{\mathfrak{p}}$, the equality and operations are given as follows, [12]:

Equality

$$
\begin{array}{ll}
w_{1}=w_{2} & \Leftrightarrow z_{11}=z_{21} \wedge z_{12}=z_{22} \\
w_{1}+w_{2} & =\left(z_{11}+z_{21}\right)+\left(z_{12}+z_{22}\right) \varepsilon \\
\lambda w_{1} & =\left(\lambda z_{11}\right)+\left(\lambda z_{12}\right) \varepsilon, \lambda \in \mathbb{R} \\
w_{1} w_{2} & =\left(z_{11} z_{21}\right)+\left(z_{11} z_{22}+z_{12} z_{21}\right) \varepsilon
\end{array}
$$

Addition

Multiplication

$\mathbb{D C}_{\mathfrak{p}}$ is a vector space over $\mathbb{R}$. A $\mathcal{D G C}$ number can be given in terms of its base elements $\{1, J, \varepsilon, \varepsilon J\}$ (or $\{1, \varepsilon, J, \varepsilon J\}$ ) as

$$
w=x_{1}+x_{2} J+y_{1} \varepsilon+y_{2} J \varepsilon,
$$

where $J$ indicates the pure generalized complex unit, $\varepsilon$ is the pure dual unit and $J \varepsilon$ is the dual-generalized complex unit. The base elements $\{1, J, \varepsilon, \varepsilon J\}$ satisfy the properties given in Table 1.

\begin{tabular}{|c||cccc|}
\hline & 1 & $J$ & $\varepsilon$ & $J \varepsilon$ \\
\hline \hline 1 & 1 & $J$ & $\varepsilon$ & $J \varepsilon$ \\
$J$ & $J$ & $\mathfrak{p}$ & $J \varepsilon$ & $\mathfrak{p} \varepsilon$ \\
$\varepsilon$ & $\varepsilon$ & $J \varepsilon$ & 0 & 0 \\
$J \varepsilon$ & $J \varepsilon$ & $\mathfrak{p} \varepsilon$ & 0 & 0 \\
\hline
\end{tabular}

Table 1. Multiplication scheme of $\mathcal{D G C}$ numbers, [12]

Moreover, the set of $\mathcal{H G C}$ and $\mathcal{C G C}$ numbers are introduced as, respectively:

$$
\mathbb{H}_{\mathbb{p}_{\mathfrak{p}}}:=\left\{w=z_{1}+z_{2} j \mid z_{1}, z_{2} \in \mathbb{C}_{\mathfrak{p}}, j^{2}=1, j \neq \pm 1, j \notin \mathbb{R}\right\},
$$

and

$$
\mathbb{C C}_{\mathfrak{p}}:=\left\{w=z_{1}+z_{2} i \mid z_{1}, z_{2} \in \mathbb{C}_{\mathfrak{p}}, i^{2}=-1, i \notin \mathbb{R}\right\}
$$

For the basis elements, we have $J j=j J$ and $J i=i J$. The operations for the $\mathcal{H G C}$ and $\mathcal{C G C}$ numbers can be given similarly (see in [12]).

We are now ready to prove our main results.

\section{$3 \quad \mathcal{D G C}$ Horadam numbers}

Let us extend the familiar Horadam number to $\mathcal{D G C}$ version.

Definition 3.1. The $\mathcal{D} \mathcal{G C}$ Horadam number $\tilde{\mathcal{W}}_{n}(a, b ; p, q)$ is defined by:

$$
\tilde{\mathcal{W}}_{n}=W_{n}+W_{n+1} J+W_{n+2} \varepsilon+W_{n+3} J \varepsilon
$$

where $W_{n}$ is the $n$-th Horadam number and $\{1, J, \varepsilon, J \varepsilon\}$ have properties given in Table 1.

These new types of Horadam numbers can be exactly examined in Table 2 . 


\begin{tabular}{|c|c|c|}
\hline$\tilde{\mathcal{W}}_{n}$ & $\tilde{\mathcal{W}}_{n}(a, b ; p, q)$ & $\mathcal{D G C}$ Horadam numbers \\
\hline$\overline{\tilde{\mathcal{F}}_{n}}$ & $\tilde{\mathcal{W}}_{n}(0,1 ; 1,-1)$ & $\mathcal{D G C}$ Fibonacci numbers [13] \\
\hline$\tilde{\mathcal{U}}_{n}$ & $\tilde{\mathcal{W}}_{n}(0,1 ; p, q)$ & $\mathcal{D G C}$ Generalized Fibonacci numbers \\
\hline$\tilde{\mathcal{L}}_{n}$ & $\tilde{\mathcal{W}}_{n}(2,1 ; 1,-1)$ & $\mathcal{D G C}$ Lucas numbers [13] \\
\hline$\tilde{\mathcal{V}}_{n}$ & $\tilde{\mathcal{W}}_{n}(2, p ; p, q)$ & $\mathcal{D G C}$ Generalized Lucas numbers \\
\hline$\tilde{\mathcal{P}}_{n}$ & $\tilde{\mathcal{W}}_{n}(0,1 ; 2,-1)$ & $\mathcal{D G C}$ Pell numbers \\
\hline$\tilde{\mathcal{P} \mathcal{L}_{n}}$ & $\tilde{\mathcal{W}}_{n}(2,2 ; 2,-1)$ & $\mathcal{D G C}$ Pell-Lucas numbers \\
\hline$\tilde{\mathcal{M}} \mathcal{P}_{n}$ & $\tilde{\mathcal{W}}_{n}(1,1 ; 2,-1)$ & $\mathcal{D G C}$ Modified Pell numbers \\
\hline$\tilde{\mathcal{J}}_{n}$ & $\tilde{\mathcal{W}}_{n}(0,1 ; 1,-2)$ & $\mathcal{D G C}$ Jacobsthal numbers \\
\hline$\tilde{\mathcal{J} \mathcal{L}_{n}}$ & $\tilde{\mathcal{W}}_{n}(2,1 ; 1,-2)$ & $\mathcal{D G C}$ Jacobsthal-Lucas numbers \\
\hline$\tilde{\mathcal{M}}_{n}$ & $\tilde{\mathcal{W}}_{n}(0,1 ; 3,2)$ & $\mathcal{D G C}$ Mersenne numbers \\
\hline$\tilde{\mathcal{T}}_{n}$ & $\tilde{\mathcal{W}}_{n}(1,3 ; 3,-2)$ & $\mathcal{D G C}$ Fermat numbers \\
\hline
\end{tabular}

Table 2. Special cases for $\mathcal{D G C}$ Horadam numbers

Definition 3.2. Let $\tilde{\mathcal{W}}_{n}, \tilde{\mathcal{W}}_{m}$ be $\mathcal{D G C}$ Horadam numbers. Then, by using the multiplication properties from Table 1, equality, addition, subtraction, scalar multiplication, and multiplication of $\mathcal{D G C}$ Horadam numbers are given, respectively, by:

$$
\begin{aligned}
\tilde{\mathcal{W}}_{n}= & \tilde{\mathcal{W}}_{m} \Leftrightarrow W_{n}=W_{m} \wedge W_{n+1}=W_{m+1} \wedge W_{n+2}=W_{m+2} \wedge W_{n+3}=W_{m+3} \\
\tilde{\mathcal{W}}_{n} \pm \tilde{\mathcal{W}}_{m}= & \left(W_{n} \pm W_{m}\right)+\left(W_{n+1} \pm W_{m+1}\right) J+\left(W_{n+2} \pm W_{m+2}\right) \varepsilon \\
& +\left(W_{n+3} \pm W_{m+3}\right) J \varepsilon \\
\lambda \tilde{\mathcal{W}}_{n}= & \lambda W_{n}+\lambda W_{n+1} J+\lambda W_{n+2} \varepsilon+\lambda W_{n+3} J \varepsilon, \lambda \in \mathbb{R} \\
\tilde{\mathcal{W}}_{n} \times \tilde{\mathcal{W}}_{m}= & W_{n} W_{m}+\mathfrak{p} W_{n+1} W_{m+1}+\left(W_{n+1} W_{m}+W_{n} W_{m+1}\right) J \\
& +\left(W_{n} W_{m+2}+W_{n+2} W_{m}+\mathfrak{p}\left(W_{n+1} W_{m+3}+W_{n+3} W_{m+1}\right)\right) \varepsilon \\
& +\left(W_{n+1} W_{m+2}+W_{n} W_{m+3}+W_{n+3} W_{m}+W_{n+2} W_{m+1}\right) J \varepsilon
\end{aligned}
$$

Remark 3.1. The $\mathcal{D G C}$ Horadam numbers satisfy the following recurrence relation

$$
\tilde{\mathcal{W}}_{n}=p \tilde{\mathcal{W}}_{n-1}-q \tilde{\mathcal{W}}_{n-2}, \quad(n \geq 2),
$$

where

$$
\tilde{\mathcal{W}}_{0}=a+b J+(p b-q a) \varepsilon+\left(p^{2} b-p q a-q b\right) J \varepsilon
$$

and

$$
\tilde{\mathcal{W}}_{1}=b+(p b-q a) J+\left(p^{2} b-p q a-q b\right) \varepsilon+\left(p^{3} b-p^{2} q a-2 p q b+q^{2} a\right) J \varepsilon,
$$

are the initial conditions. 
Proposition 3.1. The following linear recurrence relations are valid for $\mathcal{D G C}$ Horadam numbers:

- $\tilde{\mathcal{W}}_{n+2}=\left(p^{2}-q\right) \tilde{\mathcal{W}}_{n}-p q \tilde{\mathcal{W}}_{n-1}$,

- $p \tilde{\mathcal{W}}_{n+2}=\left(p^{2}-q\right) \tilde{\mathcal{W}}_{n+1}-q^{2} \tilde{\mathcal{W}}_{n-1}$,

- $\left(p^{2}-2 q\right) \tilde{\mathcal{W}}_{n+2}=q^{2} \tilde{\mathcal{W}}_{n}+\tilde{\mathcal{W}}_{n+4}$,

- $\left(p^{4}-3 p^{2} q+q^{2}\right) \tilde{\mathcal{W}}_{n+2}=\left(p^{2} q^{2}-q^{3}\right) \tilde{\mathcal{W}}_{n}+p W_{n+5}$,

- $\left(p^{4}+3 q^{2}-4 p^{2} q\right) \tilde{\mathcal{W}}_{n+2}=\left(p^{2} q^{2}-2 q^{3}\right) \tilde{\mathcal{W}}_{n}+\tilde{\mathcal{W}}_{n+6}$,

- $\left(p^{4}-5 p^{4} q+6 p^{2} q^{2}-q^{3}\right) \tilde{\mathcal{W}}_{n+2}=\left(p^{4} q^{2}-3 p^{2} q^{3}+q^{4}\right) \tilde{\mathcal{W}}_{n}+p \tilde{\mathcal{W}}_{n+7}$,

- $\tilde{\mathcal{W}}_{k+n}=\left(\alpha^{n}+\beta^{n}\right) \tilde{\mathcal{W}}_{k}-q^{n} \tilde{\mathcal{W}}_{k-n}$.

Definition 3.3. Let $\tilde{\mathcal{W}}_{n}$ be a $\mathcal{D G C}$ Horadam number. The different conjugations and modules can be defined as in Table 3:

\begin{tabular}{|l|l|}
\hline Generalized complex conjugate & $\tilde{\mathcal{W}}_{n}^{\dagger_{1}}=\left(W_{n}-W_{n+1} J\right)+\left(W_{n+2}-W_{n+3} J\right) \varepsilon$ \\
Generalized complex module & $\mathrm{N}_{\tilde{\mathcal{W}}_{n}}^{\dagger_{1}}=\tilde{\mathcal{W}}_{n} \times \tilde{\mathcal{W}}_{n}^{\dagger_{1}}$ \\
Dual conjugate & $\tilde{\mathcal{W}}_{n}^{\dagger_{2}}=\left(W_{n}+W_{n+1} J\right)-\left(W_{n+2}+W_{n+3} J\right) \varepsilon$ \\
Dual module & $\mathrm{N}_{\tilde{\mathcal{W}}_{n}}^{\dagger_{2}}=\tilde{\mathcal{W}}_{n} \times \tilde{\mathcal{W}}_{n}^{\dagger_{2}}$ \\
Coupled conjugate & $\tilde{\mathcal{W}}_{n}^{\dagger_{3}}=\left(W_{n}-W_{n+1} J\right)-\left(W_{n+2}-W_{n+3} J\right) \varepsilon$ \\
Coupled module & $\mathrm{N}_{\tilde{\mathcal{W}}_{n}}^{\dagger_{3}}=\tilde{\mathcal{W}}_{n} \times \tilde{\mathcal{W}}_{n}^{\dagger_{3}}$ \\
Anti-dual conjugate & $\tilde{\mathcal{W}}_{n}^{\dagger_{4}}=\left(W_{n+2}+W_{n+3} J\right)-\left(W_{n}+W_{n+1} J\right) \varepsilon$ \\
\hline
\end{tabular}

Table 3. Conjugations and modules of $\mathcal{D G C}$ Horadam numbers

Proposition 3.2. Let $\tilde{\mathcal{W}}_{n}$ be $\mathcal{D} \mathcal{G C}$ Horadam number. Then, the below properties can be given:

- $\tilde{\mathcal{W}}_{n}+\tilde{\mathcal{W}}_{n}^{\dagger_{1}}=2\left(W_{n}+W_{n+2} \varepsilon\right)$

- $\tilde{\mathcal{W}}_{n} \times \tilde{\mathcal{W}}_{n}^{\dagger_{1}}=W_{n}^{2}-\mathfrak{p} W_{n+1}^{2}+2\left(W_{n} W_{n+2}-\mathfrak{p} W_{n+1} W_{n+3}\right) \varepsilon$,

- $\tilde{\mathcal{W}}_{n}+\tilde{\mathcal{W}}_{n}^{\dagger}=2\left(W_{n}+W_{n+1} J\right)$,

- $\tilde{\mathcal{W}}_{n} \times \tilde{\mathcal{W}}_{n}^{\dagger_{2}}=W_{n}^{2}+\mathfrak{p} W_{n+1}^{2}+2 W_{n} W_{n+1} J$,

- $\tilde{\mathcal{W}}_{n}+\tilde{\mathcal{W}}_{n}^{\dagger_{3}}=2\left(W_{n}+W_{n+3} J \varepsilon\right)$,

- $\tilde{\mathcal{W}}_{n} \times \tilde{\mathcal{W}}_{n}^{\dagger_{3}}=W_{n}^{2}-\mathfrak{p} W_{n+1}^{2}+2\left(W_{n} W_{n+3}-W_{n+1} W_{n+2}\right) J \varepsilon$,

- $\tilde{\mathcal{W}}_{n}-\varepsilon \tilde{\mathcal{W}}_{n}^{\dagger_{4}}=W_{n}+W_{n+1} J$,

- $\varepsilon \tilde{\mathcal{W}}_{n}+\tilde{\mathcal{W}}_{n}^{\dagger_{4}}=W_{n+2}+W_{n+3} J$. 


\subsection{Fundamental formulas for $\mathcal{D G C}$ Horadam numbers}

We also seek to identify universal identities.

Theorem 3.1. Let $\tilde{\mathcal{W}}_{0}, \tilde{\mathcal{W}}_{1}$ be initial $\mathcal{D G C}$ Horadam numbers. Then, the generating function of $\mathcal{D G C}$ Horadam numbers is as follows:

$$
\tilde{g}(x)=\sum_{n=0}^{\infty} \tilde{\mathcal{W}}_{n} x^{n}=\frac{\tilde{\mathcal{W}}_{0}+\left(\tilde{\mathcal{W}}_{1}-p \tilde{\mathcal{W}}_{0}\right) x}{1-p x+q x^{2}}
$$

Proof. The proof is straightforward by using equation (4).

Let us give the following theorems related to well-known identities for Horadam numbers where $\tilde{\alpha}^{*}=1+\alpha J+\alpha^{2} \varepsilon+\alpha^{3} J \varepsilon, \tilde{\beta}^{*}=1+\beta J+\beta^{2} \varepsilon+\beta^{3} J \varepsilon$ [13] and $A=\frac{b-a \beta}{\alpha-\beta}$, $B=\frac{a \alpha-b}{\alpha-\beta},[18]$.

Theorem 3.2. Let $\tilde{\mathcal{W}}_{n}$ be $\mathcal{D} \mathcal{G C}$ Horadam number. Then, for $n \geq 2$, the Binet's formula can be written as follows:

$$
\tilde{\mathcal{W}}_{n}=A \tilde{\alpha}^{*} \alpha^{n}+B \tilde{\beta}^{*} \beta^{n} .
$$

Proof. By using (3), the Binet's formula for $\mathcal{D G C}$ Horadam numbers can be calculated as below:

$$
\begin{aligned}
\tilde{\mathcal{W}}_{n} & =A \alpha^{n}+B \beta^{n}+\left(A \alpha^{n+1}+B \beta^{n+1}\right) J+\left(A \alpha^{n+2}+B \beta^{n+2}\right) \varepsilon+\left(A \alpha^{n+3} B \beta^{n+3}\right) J \varepsilon \\
& =A \alpha^{n}\left(1+\alpha J+\alpha^{2} \varepsilon+\alpha^{3} J \varepsilon\right)+B \beta^{n}\left(1+\beta J+\beta^{2} \varepsilon+\beta^{3} J \varepsilon\right) \\
& =A \tilde{\alpha}^{*} \alpha^{n}+B \tilde{\beta}^{*} \beta^{n} .
\end{aligned}
$$

The preceding theorem allows us to give the following corollary and theorems.

Corollary 3.1. Let $\tilde{\mathcal{W}}_{-n}$ be a nega $\mathcal{D G C}$ Horadam number $(\mathcal{D G C}$ Horadam number with negative subscript). Then, the following equality holds:

$$
\tilde{\mathcal{W}}_{-n}=A \tilde{\alpha}^{*} \alpha^{-n}+B \tilde{\beta}^{*} \beta^{-n} .
$$

Theorem 3.3. The exponential generating function of $\mathcal{D G C}$ Horadam numbers is as follows:

$$
\sum_{n=0}^{\infty} \tilde{\mathcal{W}}_{n} \frac{x^{n}}{n !}=A \tilde{\alpha}^{*} e^{\alpha x}+B \tilde{\beta}^{*} e^{\beta x} .
$$

Proof. Considering equation (13), the proof is straightforward.

Inspired to the study Theorem 3 in [22], we can give the following theorem:

Theorem 3.4. Let $c$, $d$ and $r$ be nonzero integers. For $n \geq 0$,

$$
\tilde{\mathcal{W}}_{c n+r}=\sum_{k=0}^{n}\left(\begin{array}{l}
n \\
k
\end{array}\right) t^{n-k} s^{k} \tilde{\mathcal{W}}_{d k+r}
$$

if and only if $s=\frac{\alpha^{c}-\beta^{c}}{\alpha^{d}-\beta^{d}}$ and $t=q^{c} \frac{\alpha^{d-c}-\beta^{d-c}}{\alpha^{d}-\beta^{d}}$.

Proof. The proof is completed simply using equation (2) and equation (15). 
Theorem 3.5. Let $\tilde{\mathcal{W}}_{n}, \tilde{\mathcal{W}}_{m}$ be $\mathcal{D} \mathcal{G C}$ Horadam numbers. Then, the D'Ocagne's identity for $\mathcal{D G C}$ Horadam numbers is:

$$
\tilde{\mathcal{W}}_{m} \times \tilde{\mathcal{W}}_{n+1}-\tilde{\mathcal{W}}_{m+1} \times \tilde{\mathcal{W}}_{n}=-A B \tilde{\alpha}^{*} \tilde{\beta}^{*} \sqrt{p^{2}-4 q}\left(\alpha^{m} \beta^{n}-\alpha^{n} \beta^{m}\right)
$$

Proof. With the aid of the Binet's formula for $\mathcal{D G C}$ Horadam numbers given in equation (13), we can assert that

$$
\tilde{\mathcal{W}}_{m} \times \tilde{\mathcal{W}}_{n+1}-\tilde{\mathcal{W}}_{m+1} \times \tilde{\mathcal{W}}_{n}=-A B \tilde{\alpha}^{*} \tilde{\beta}^{*}(\alpha-\beta)\left(\alpha^{m} \beta^{n}-\alpha^{n} \beta^{m}\right)
$$

By substituting equation (2) into (17), the proof is completed.

Theorem 3.6. Let $\tilde{\mathcal{W}}_{n}$ be $\mathcal{D} \mathcal{G C}$ Horadam number. Then, the Catalan's identity for $\mathcal{D G C}$ Horadam numbers is:

$$
\tilde{\mathcal{W}}_{n}^{2}-\tilde{\mathcal{W}}_{n+r} \times \tilde{\mathcal{W}}_{n-r}=-A B \tilde{\alpha}^{*} \tilde{\beta}^{*} q^{n-r}\left(\alpha^{r}-\beta^{r}\right)^{2}
$$

Proof. Similar to the previous proof, by using equation (13), we can write that

$$
\begin{aligned}
\tilde{\mathcal{W}}_{n}^{2}-\tilde{\mathcal{W}}_{n+r} \times \tilde{\mathcal{W}}_{n-r} & =A B \tilde{\alpha}^{*} \tilde{\beta}^{*}\left(2 \alpha^{n} \beta^{n}-\alpha^{n+r} \beta^{n-r}-\alpha^{n-r} \beta^{n+r}\right) \\
& =-A B \tilde{\alpha}^{*} \tilde{\beta}^{*}(\alpha \beta)^{n-r}\left(-2(\alpha \beta)^{r}+\alpha^{2 r}+\beta^{2 r}\right) .
\end{aligned}
$$

Substituting equality (2) into (19), we have equation (18).

Theorem 3.7. Let $\tilde{\mathcal{W}}_{n}$ be $\mathcal{D} \mathcal{G C}$ Horadam number. Then, the Cassini's identity for $\mathcal{D G C}$ Horadam numbers is:

$$
\tilde{\mathcal{W}}_{n}^{2}-\tilde{\mathcal{W}}_{n+1} \times \tilde{\mathcal{W}}_{n-1}=-A B \tilde{\alpha}^{*} \tilde{\beta}^{*} q^{n-1}\left(p^{2}-4 q\right)
$$

Proof. By taking $r=1$ in the Catalan's identity given in equation (18) and considering equalities given in equation (2), the Cassini's identity for $\mathcal{D G C}$ Horadam numbers can be obtained.

\subsection{Matrix representations of $\mathcal{D G C}$ Horadam numbers}

A natural question to ask is if matrix representation of dual, generalized and real numbers, can be generalized for $\mathcal{D G C}$ Horadam Numbers.

Theorem 3.8. Every $\tilde{\mathcal{W}}_{n}=\left(W_{n}+W_{n+1} J\right)+\left(W_{n+2}+W_{n+3} J\right) \varepsilon$ can be represented by the following $2 \times 2$ matrix:

$$
\mathcal{A}_{\tilde{\mathcal{W}}_{n}}=\left[\begin{array}{cc}
W_{n}+W_{n+1} J & 0 \\
W_{n+2}+W_{n+3} J & W_{n}+W_{n+1} J
\end{array}\right] .
$$

Proof. The main idea of the proof is to take an isomorphism $\mathcal{A}$ between dual numbers and $2 \times 2$ matrices. Here, $\mathcal{A}$ is a linear transformation between $\mathcal{D G C}$ Horadam numbers and the matrices

$$
\left\{\left[\begin{array}{cc}
W_{n}+W_{n+1} J & 0 \\
W_{n+2}+W_{n+3} J & W_{n}+W_{n+1} J
\end{array}\right] \mid W_{n} \text { is the } n \text {-th Horadam number }\right\} .
$$

The columns of the matrix $\mathcal{A}_{\tilde{\mathcal{W}}_{n}}$ are represented by the coefficients of the elements $\left\{\tilde{\mathcal{W}}_{n}, \tilde{\mathcal{W}}_{n} \varepsilon\right\}$, considered in respect to the basis $\{1, \varepsilon\}$. Hence, $\mathcal{D} \mathcal{G C}$ Horadam numbers is the subset of $2 \times 2$ matrices with generalized complex Horadam numbers. 
Theorem 3.9. Every $\tilde{\mathcal{W}}_{n}=W_{n}+W_{n+1} J+W_{n+2}+W_{n+3} J \varepsilon$ can be also represented by a matrix in $\mathcal{M}_{4}(\mathbb{R})$ :

$$
\mathcal{B}_{\tilde{\mathcal{W}}_{n}}=\left[\begin{array}{cccc}
W_{n} & \mathfrak{p} W_{n+1} & 0 & 0 \\
W_{n+1} & W_{n} & 0 & 0 \\
W_{n+2} & \mathfrak{p} W_{n+3} & W_{n} & \mathfrak{p} W_{n+1} \\
W_{n+3} & W_{n+2} & W_{n+1} & W_{n}
\end{array}\right]
$$

Proof. With the linear transformation $\Lambda\left(W_{n}+W_{n+1} J\right)=\left[\begin{array}{cc}W_{n} & \mathfrak{p} W_{n+1} \\ W_{n+1} & W_{n}\end{array}\right]$, real Horadam matrix representation is given by:

$$
\mathcal{B}_{\tilde{\mathcal{W}}_{n}}=\left[\begin{array}{cc}
\Lambda\left(W_{n}+W_{n+1} J\right) & \Lambda(0) \\
\Lambda\left(W_{n+2}+W_{n+3} J\right) & \Lambda\left(W_{n}+W_{n+1} J\right)
\end{array}\right]=\left[\begin{array}{cccc}
W_{n} & \mathfrak{p} W_{n+1} & 0 & 0 \\
W_{n+1} & W_{n} & 0 & 0 \\
W_{n+2} & \mathfrak{p} W_{n+3} & W_{n} & \mathfrak{p} W_{n+1} \\
W_{n+3} & W_{n+2} & W_{n+1} & W_{n}
\end{array}\right] .
$$

The columns of the matrix $\mathcal{B}_{\tilde{\mathcal{W}}_{n}}$ are represented by the coefficients of the elements $\left\{\tilde{\mathcal{W}}_{n}, \tilde{\mathcal{W}}_{n} J, \tilde{\mathcal{W}}_{n} \varepsilon, \tilde{\mathcal{W}}_{n} J \varepsilon\right\}$, considered in respect to the basis $\{1, J, \varepsilon, J \varepsilon\}$. Moreover, $\mathcal{D G C}$ Horadam number is the subset of $4 \times 4$ matrices with real Horadam numbers.

Corollary 3.2. $\mathcal{B}_{\tilde{\mathcal{W}}_{n}}$ can be written also in the form:

$\mathcal{B}_{\tilde{\mathcal{W}}_{n}}=W_{n} I_{4}+W_{n+1} \mathcal{J}+W_{n+2} \mathcal{E}+W_{n+3} \mathcal{J E}$,
where $\mathcal{B}_{J}=\mathcal{J}=\left[\begin{array}{cccc}0 & \mathfrak{p} & 0 & 0 \\ 1 & 0 & 0 & 0 \\ 0 & 0 & 0 & \mathfrak{p} \\ 0 & 0 & 1 & 0\end{array}\right], \mathcal{B}_{\varepsilon}=\mathcal{E}=\left[\begin{array}{llll}0 & 0 & 0 & 0 \\ 0 & 0 & 0 & 0 \\ 1 & 0 & 0 & 0 \\ 0 & 1 & 0 & 0\end{array}\right], \mathcal{B}_{J \varepsilon}=\mathcal{J} \mathcal{E}=\left[\begin{array}{llll}0 & 0 & 0 & 0 \\ 0 & 0 & 0 & 0 \\ 0 & \mathfrak{p} & 0 & 0 \\ 1 & 0 & 0 & 0\end{array}\right]$.

Theorem 3.10. For any $\tilde{\mathcal{W}}_{n}, \tilde{\mathcal{W}}_{m}$ and $\lambda \in \mathbb{R}$, the following properties hold:

- $\mathcal{A}_{\lambda \tilde{\mathcal{W}}_{n}}=\lambda \mathcal{A}_{\tilde{\mathcal{W}}_{n}}$,

- $\mathcal{B}_{\lambda \tilde{\mathcal{W}}_{n}}=\lambda \mathcal{B}_{\tilde{\mathcal{W}}_{n}}$,

- $\mathcal{A}_{\tilde{\mathcal{W}}_{n} \tilde{\mathcal{W}}_{m}}=\mathcal{A}_{\tilde{\mathcal{W}}_{n}} \mathcal{A}_{\tilde{\mathcal{W}}_{m}}$,

- $\mathcal{B}_{\tilde{\mathcal{W}}_{n} \tilde{\mathcal{W}}_{m}}=\mathcal{B}_{\tilde{\mathcal{W}}_{n}} \mathcal{B}_{\tilde{\mathcal{W}}_{m}}$,

- $\operatorname{det}\left(\mathcal{A}_{\tilde{\mathcal{W}}_{n}}\right)=\left(W_{n}+W_{n+1} J\right)^{2}$,

- $\operatorname{det}\left(\mathcal{B}_{\tilde{\mathcal{W}}_{n}}\right)=\left(W_{n}^{2}-\mathfrak{p} W_{n+1}^{2}\right)^{2}$.

According to Theorem 3.9, the following corollary is immediate.

Corollary 3.3. The column matrix representation of an arbitrary $\mathcal{D G C}$ Horadam number $\tilde{\mathcal{W}}_{n}$ with respect to the standard basis is merely the collection of its coefficients:

$$
\tilde{\mathcal{W}}_{n}=\left[\begin{array}{llll}
W_{n} & W_{n+1} & W_{n+2} & W_{n+3}
\end{array}\right]^{T} .
$$

The multiplication of $\tilde{\mathcal{W}}_{n}$ and $\tilde{\mathcal{W}}_{m}$ can also be given as 


$$
\tilde{\mathcal{W}}_{n} \times \tilde{\mathcal{W}}_{m}=\left[\begin{array}{cccc}
W_{n} & \mathfrak{p} W_{n+1} & 0 & 0 \\
W_{n+1} & W_{n} & 0 & 0 \\
W_{n+2} & \mathfrak{p} W_{n+3} & W_{n} & \mathfrak{p} W_{n+1} \\
W_{n+3} & W_{n+2} & W_{n+1} & W_{n}
\end{array}\right]\left[\begin{array}{c}
W_{m} \\
W_{m+1} \\
W_{m+2} \\
W_{m+3}
\end{array}\right] .
$$

So, we can say that the multiplication of $\mathcal{D G C}$ Horadam numbers can be calculated by matrix product.

Theorem 3.11. Let $\tilde{\mathcal{W}}_{n}=W_{n}+W_{n+1} J+W_{n+2} \varepsilon+W_{n+3} J \varepsilon$. Then,

- $\rho \tilde{\mathcal{W}}_{n}=\tilde{\mathcal{W}}_{n}^{\dagger}$,

- $\sigma \tilde{\mathcal{W}}_{n}=\tilde{\mathcal{W}}_{n}^{\dagger}$,

- $\tau \tilde{\mathcal{W}}_{n}=\tilde{\mathcal{W}}_{n}^{\dagger}$,

where $\rho=\operatorname{diag}(1,-1,1,-1), \sigma=\operatorname{diag}(1,1,-1,-1), \tau=\operatorname{diag}(1,-1,-1,1) \in \mathcal{M}_{4}(\mathbb{R})$.

\section{$4 \quad \mathcal{H} \mathcal{G C}$ and $\mathcal{C G C}$ Horadam numbers}

With a thought similar to Section 3, algebraic properties, linear recurrence relations, generating function, well-known formulas and matrix forms for $\mathcal{H G C}$ and $\mathcal{C G C}$ Horadam numbers are investigated in this section.

\section{1 $\mathcal{H} \mathcal{G C}$ Horadam numbers}

Definition 4.1. The $\mathcal{H G C}$ Horadam number $\hat{\mathcal{W}}_{n}(a, b ; p, q)$ is defined as:

$$
\hat{\mathcal{W}}_{n}(a, b ; p, q)=W_{n}+W_{n+1} J+W_{n+2} j+W_{n+3} J j,
$$

where $W_{n}$ is the $n$-th Horadam number and the set $\{1, J, j, J j\}$ has multiplicative properties given in [12].

Remark 4.1. The $\mathcal{H G C}$ Horadam numbers satisfy the recurrence relation

$$
\hat{\mathcal{W}}_{n}=p \hat{\mathcal{W}}_{n-1}-q \hat{\mathcal{W}}_{n-2}, \quad(n \geq 2),
$$

with initial conditions ${ }^{2} \hat{\mathcal{W}}_{0}$ and $\hat{\mathcal{W}}_{1}$.

Linear recurrence relations, different conjugations and modules, and several properties can also be obtained for the $\mathcal{H C C}$ Horadam numbers similiar to Proposition 3.1, Definition 3.3 and Proposition 3.2, respectively.

Theorem 4.1. Let $\hat{\mathcal{W}}_{n}, \hat{\mathcal{W}}_{m}$ be $\mathcal{H C C}$ Horadam numbers and $\hat{\alpha}^{*}=1+\alpha J+\alpha^{2} j+\alpha^{3} J j$, $\hat{\beta}^{*}=1+\beta J+\beta^{2} j+\beta^{3} J j$. Then, the following identities hold:

$$
\text { Binet's formula: } \quad \hat{\mathcal{W}}_{n}=A \hat{\alpha}^{*} \alpha^{n}+B \hat{\beta}^{*} \beta^{n} \text {. }
$$

D'Ocagne's identity: $\quad \hat{\mathcal{W}}_{m} \times \hat{\mathcal{W}}_{n+1}-\hat{\mathcal{W}}_{m+1} \times \hat{\mathcal{W}}_{n}=-A B \hat{\alpha}^{*} \hat{\beta}^{*} \sqrt{p^{2}-4 q}\left(\alpha^{m} \beta^{n}-\alpha^{n} \beta^{m}\right)$.

Catalan's identity: $\quad \hat{\mathcal{W}}_{n}^{2}-\hat{\mathcal{W}}_{n+r} \times \hat{\mathcal{W}}_{n-r}=-A B \hat{\alpha}^{*} \hat{\beta}^{*} q^{n-r}\left(\alpha^{r}-\beta^{r}\right)^{2}$.

Cassini's identity: $\quad \hat{\mathcal{W}}_{n}^{2}-\hat{\mathcal{W}}_{n+1} \times \hat{\mathcal{W}}_{n-1}=-A B \hat{\alpha}^{*} \hat{\beta}^{*} q^{n-1}\left(p^{2}-4 q\right)$.

\footnotetext{
${ }^{2} \hat{\mathcal{W}}_{0}$ and $\hat{\mathcal{W}}_{1}$ can easily be calculated similarly to the equations (10) and (11).
} 
Theorem 4.2. The matrix representations of $\hat{\mathcal{W}}_{n}$ with respect to the bases $\{1, J, j, J j\}$ and $\{1, j\}$ are, respectively,

$$
\begin{aligned}
\mathcal{C}_{\hat{\mathcal{W}}_{n}}= & {\left[\begin{array}{ccc}
W_{n}+W_{n+1} J & W_{n+2}+W_{n+3} J \\
W_{n+2}+W_{n+3} J & W_{n}+W_{n+1} J
\end{array}\right], } \\
\mathcal{D}_{\hat{\mathcal{W}}_{n}}= & {\left[\begin{array}{cccc}
W_{n} & \mathfrak{p} W_{n+1} & W_{n+2} & \mathfrak{p} W_{n+3} \\
W_{n+1} & W_{n} & W_{n+3} & W_{n+2} \\
W_{n+2} & \mathfrak{p} W_{n+3} & W_{n} & \mathfrak{p} W_{n+1} \\
W_{n+3} & W_{n+2} & W_{n+1} & W_{n}
\end{array}\right] . }
\end{aligned}
$$

Corollary 4.1. $\mathcal{D}_{\hat{\mathcal{W}}_{n}}$ can be written also in the form:

$\mathcal{D}_{\hat{\mathcal{V}}_{n}}=W_{n} I_{4}+W_{n+1} \mathcal{J}+W_{n+2} \mathfrak{j}+W_{n+3} \mathcal{J j}$,
where $\mathcal{D}_{J}=\mathcal{J}=\left[\begin{array}{llll}0 & \mathfrak{p} & 0 & 0 \\ 1 & 0 & 0 & 0 \\ 0 & 0 & 0 & \mathfrak{p} \\ 0 & 0 & 1 & 0\end{array}\right], \mathcal{D}_{j}=\mathfrak{j}=\left[\begin{array}{llll}0 & 0 & 1 & 0 \\ 0 & 0 & 0 & 1 \\ 1 & 0 & 0 & 0 \\ 0 & 1 & 0 & 0\end{array}\right], \mathcal{D}_{J j}=\mathcal{J j}=\left[\begin{array}{llll}0 & 0 & 0 & \mathfrak{p} \\ 0 & 0 & 1 & 0 \\ 0 & \mathfrak{p} & 0 & 0 \\ 1 & 0 & 0 & 0\end{array}\right]$.

Corollary 4.2. The multiplication of $\hat{\mathcal{W}}_{n}$ and $\hat{\mathcal{W}}_{m}$ can also be calculated as:

$$
\hat{\mathcal{W}}_{n} \times \hat{\mathcal{W}}_{m}=\left[\begin{array}{cccc}
W_{n} & \mathfrak{p} W_{n+1} & W_{n} & \mathfrak{p} W_{n+3} \\
W_{n+1} & W_{n} & W_{n+3} & W_{n+2} \\
W_{n+2} & \mathfrak{p} W_{n+3} & W_{n} & \mathfrak{p} W_{n+1} \\
W_{n+3} & W_{n+2} & W_{n+1} & W_{n}
\end{array}\right]\left[\begin{array}{c}
W_{m} \\
W_{m+1} \\
W_{m+2} \\
W_{m+3}
\end{array}\right]
$$

Theorem 4.3. For $\hat{\mathcal{W}}_{n}$ and $\rho=\operatorname{diag}(1,-1,1,-1), \sigma=\operatorname{diag}(1,1,-1,-1), \tau=\operatorname{diag}(1,-1,-1,1) \in$ $\mathcal{M}_{4}(\mathbb{R})$, the following equalities are satisfied:

- $\rho \hat{\mathcal{W}}_{n}=\hat{\mathcal{W}}_{n}^{\dagger}$,

- $\sigma \hat{\mathcal{W}}_{n}=\hat{\mathcal{W}}_{n}^{\dagger_{2}}$,

- $\tau \hat{\mathcal{W}}_{n}=\hat{\mathcal{W}}_{n}^{\dagger_{3}}$.

\section{2 $\mathcal{C} \mathcal{G C}$ Horadam numbers}

This section describes $\mathcal{C G C}$ Horadam numbers and some key relations.

Definition 4.2. The $\mathcal{H G C}$ Horadam number $\overline{\mathcal{W}}_{n}(a, b ; p, q)$ is defined as:

$$
\overline{\mathcal{W}}_{n}=W_{n}+W_{n+1} J+W_{n+2} i+W_{n+3} J i
$$

where $W_{n}$ is the $n$-th Horadam number and the set $\{1, J, i, J i\}$ has multiplicative properties given in [12].

Remark 4.2. The $\mathcal{C G C}$ Horadam numbers satisfy the recurrence relation

$$
\overline{\mathcal{W}}_{n}=p \overline{\mathcal{W}}_{n-1}-q \overline{\mathcal{W}}_{n-2}, \quad(n \geq 2),
$$

with initial conditions ${ }^{3} \overline{\mathcal{W}}_{0}$ and $\overline{\mathcal{W}}_{1}$.

\footnotetext{
${ }^{3} \overline{\mathcal{W}}_{0}$ and $\overline{\mathcal{W}}_{1}$ can easily be calculated similarly to the equations (10) and (11).
} 
Several features for the $\mathcal{C G C}$ Horadam numbers can be observed by taking into account the way given in Proposition 3.1, Definition 3.3 and Proposition 3.2.

Theorem 4.4. Let $\overline{\mathcal{W}}_{n}, \overline{\mathcal{W}}_{m}$ be $\mathcal{C G C}$ Horadam numbers and $\bar{\alpha}^{*}=1+\alpha J+\alpha^{2} i+\alpha^{3} J i$, $\bar{\beta}^{*}=1+\beta J+\beta^{2} i+\beta^{3} J i$. Then, the following identities hold:

Binet's formula: $\quad \overline{\mathcal{W}}_{n}=A \bar{\alpha}^{*} \alpha^{n}+B \bar{\beta}^{*} \beta^{n}$.

D'Ocagne's identity: $\quad \overline{\mathcal{W}}_{m} \times \overline{\mathcal{W}}_{n+1}-\overline{\mathcal{W}}_{m+1} \times \overline{\mathcal{W}}_{n}=-A B \bar{\alpha}^{*} \bar{\beta}^{*} \sqrt{p^{2}-4 q}\left(\alpha^{m} \beta^{n}-\alpha^{n} \beta^{m}\right)$.

Catalan's identity: $\quad \overline{\mathcal{W}}_{n}^{2}-\overline{\mathcal{W}}_{n+r} \times \overline{\mathcal{W}}_{n-r}=-A B \bar{\alpha}^{*} \bar{\beta}^{*} q^{n-r}\left(\alpha^{r}-\beta^{r}\right)^{2}$.

Cassini's identity: $\quad \overline{\mathcal{W}}_{n}^{2}-\overline{\mathcal{W}}_{n+1} \times \overline{\mathcal{W}}_{n-1}=-A B \bar{\alpha}^{*} \bar{\beta}^{*} q^{n-1}\left(p^{2}-4 q\right)$.

Theorem 4.5. The matrix representations of $\overline{\mathcal{W}}_{n}$ with respect to the bases $\{1, J, i, J i\}$ and $\{1, i\}$ are, respectively,

$$
\begin{gathered}
\mathcal{X}_{\overline{\mathcal{W}}_{n}}=\left[\begin{array}{crc}
W_{n}+W_{n+1} J & -\left(W_{n+2}+W_{n+3} J\right) \\
W_{n+2}+W_{n+3} J & W_{n}+W_{n+1} J
\end{array}\right], \\
\mathcal{Y}_{\overline{\mathcal{W}}_{n}}=\left[\begin{array}{cccc}
W_{n} & \mathfrak{p} W_{n+1} & -W_{n+2} & -\mathfrak{p} W_{n+3} \\
W_{n+1} & W_{n} & -W_{n+3} & -W_{n+2} \\
W_{n+2} & \mathfrak{p} W_{n+3} & W_{n} & \mathfrak{p} W_{n+1} \\
W_{n+3} & W_{n+2} & W_{n+1} & W_{n}
\end{array}\right] .
\end{gathered}
$$

Corollary 4.3. $\mathcal{Y}_{\overline{\mathcal{W}}_{n}}$ can be written also in the form:

$$
\mathcal{Y}_{\overline{\mathcal{W}}_{n}}=W_{n} I_{4}+W_{n+1} \mathcal{J}+W_{n+2} \mathcal{I}+W_{n+3} \mathcal{J} \mathcal{I}
$$

where $\mathcal{Y}_{J}=\mathcal{J}=\left[\begin{array}{llll}0 & \mathfrak{p} & 0 & 0 \\ 1 & 0 & 0 & 0 \\ 0 & 0 & 0 & \mathfrak{p} \\ 0 & 0 & 1 & 0\end{array}\right], \mathcal{Y}_{i}=\mathcal{I}=\left[\begin{array}{rrrr}0 & 0 & -1 & 0 \\ 0 & 0 & 0 & -1 \\ 1 & 0 & 0 & 0 \\ 0 & 1 & 0 & 0\end{array}\right], \mathcal{Y}_{J i}=\mathcal{J} \mathcal{I}=\left[\begin{array}{rrrr}0 & 0 & 0 & -\mathfrak{p} \\ 0 & 0 & -1 & 0 \\ 0 & \mathfrak{p} & 0 & 0 \\ 1 & 0 & 0 & 0\end{array}\right]$.

Corollary 4.4. The multiplication of $\overline{\mathcal{W}}_{n}$ and $\overline{\mathcal{W}}_{m}$ can also be obtained as

$$
\overline{\mathcal{W}}_{n} \times \overline{\mathcal{W}}_{m}=\left[\begin{array}{cccc}
W_{n} & \mathfrak{p} W_{n+1} & -W_{n+2} & -\mathfrak{p} W_{n+3} \\
W_{n+1} & W_{n} & -W_{n+3} & -W_{n+2} \\
W_{n+2} & \mathfrak{p} W_{n+3} & W_{n} & \mathfrak{p} W_{n+1} \\
W_{n+3} & W_{n+2} & W_{n+1} & W_{n}
\end{array}\right]\left[\begin{array}{c}
W_{m} \\
W_{m+1} \\
W_{m+2} \\
W_{m+3}
\end{array}\right]
$$

Theorem 4.6. For $\overline{\mathcal{W}}_{n}$ and $\rho=\operatorname{diag}(1,-1,1,-1), \sigma=\operatorname{diag}(1,1,-1,-1), \tau=\operatorname{diag}(1,-1,-1,1) \in$ $\mathcal{M}_{4}(\mathbb{R})$, the following equalities hold:

- $\rho \overline{\mathcal{W}}_{n}=\overline{\mathcal{W}}_{n}^{\dagger}$,

- $\sigma \overline{\mathcal{W}}_{n}=\overline{\mathcal{W}}_{n}^{\dagger_{2}}$,

- $\tau \overline{\mathcal{W}}_{n}=\overline{\mathcal{W}}_{n}^{\dagger}$. 


\section{Computational results}

The following examples demonstrate the above results.

Example 5.1. The fundamental identities hold for given values:

- D'Ocagne's identity for $\mathcal{D G C}$ Jacobsthal numbers:

$$
\tilde{\mathcal{J}}_{3} \times \tilde{\mathcal{J}}_{6}-\tilde{\mathcal{J}}_{4} \times \tilde{\mathcal{J}}_{5}=8[(1-\mathfrak{p} 2)+J+5(1-\mathfrak{p} 2) \varepsilon+5 J \varepsilon],
$$

where $m=3, n=5$ and $\tilde{\mathcal{W}}_{5}(0,1 ; 1,-2)=\tilde{\mathcal{J}}_{5}$.

- Catalan's identity for $\mathcal{H G C}$ Pell numbers:

$$
\hat{\mathcal{P}}_{2}^{2}-\hat{\mathcal{P}}_{3} \times \hat{\mathcal{P}}_{1}=-2[(1-\mathfrak{p})+2 J+3(1-\mathfrak{p}) j+6 J j],
$$

where $n=2, r=1$ and $\hat{\mathcal{W}}_{2}(0,1 ; 2,-1)=\hat{\mathcal{P}}_{2}$.

- Cassini's identity for $\mathcal{C G C}$ Lucas numbers:

$$
\overline{\mathcal{L}}_{3}^{2}-\overline{\mathcal{L}}_{4} \times \overline{\mathcal{L}}_{2}=-15[(1-\mathfrak{p}) i+J i],
$$

where $n=3$ and $\overline{\mathcal{W}}_{3}(2,1 ; 1,-1)=\overline{\mathcal{L}}_{3}$.

Example 5.2. The following observations can be given for

- $\tilde{\mathcal{W}}_{2}(2,1 ; 1,-2)=\tilde{\mathcal{J}} \mathcal{L}_{2}, \mathcal{D G C}$ Jacobsthal-Lucas numbers:

$$
\begin{aligned}
\mathcal{A}_{\tilde{\mathcal{W}}_{2}}= & {\left[\begin{array}{cc}
5+7 J & 0 \\
17+31 J & 5+7 J
\end{array}\right], \quad \operatorname{det} \mathcal{A}_{\tilde{\mathcal{W}}_{2}}=25+\mathfrak{p} 49+70 J, } \\
\mathcal{B}_{\tilde{\mathcal{W}}_{2}}= & {\left[\begin{array}{llll}
5 & \mathfrak{p} 7 & 0 & 0 \\
7 & 5 & 0 & 0 \\
17 & \mathfrak{p} 31 & 5 & \mathfrak{p} 7 \\
31 & 17 & 7 & 5
\end{array}\right], \quad \operatorname{det} \mathcal{B}_{\tilde{\mathcal{W}}_{2}}=\mathfrak{p}^{2} 2401-\mathfrak{p} 2450+625, }
\end{aligned}
$$

and

$$
\tilde{\mathcal{W}}_{2}^{\dagger_{2}}=\sigma \tilde{\mathcal{W}}_{2}=\left[\begin{array}{cccc}
1 & 0 & 0 & 0 \\
0 & 1 & 0 & 0 \\
0 & 0 & -1 & 0 \\
0 & 0 & 0 & -1
\end{array}\right]\left[\begin{array}{c}
5 \\
7 \\
17 \\
31
\end{array}\right]=\left[\begin{array}{cccc}
5 & 7 & -17 & -31
\end{array}\right]^{T}
$$

- $\hat{\mathcal{W}}_{2}(2,2 ; 2,-1)=\hat{\mathcal{P L}}_{2}, \mathcal{H G C}$ Pell-Lucas numbers:

$$
\begin{aligned}
\mathcal{C}_{\hat{\mathcal{W}}_{2}}= & {\left[\begin{array}{cc}
6+14 J & 34+82 J \\
34+82 J & 6+14 J
\end{array}\right], \quad \operatorname{det} \mathcal{C}_{\hat{\mathcal{W}}_{2}}=-1120-\mathfrak{p} 6528-5408 J, } \\
\mathcal{D}_{\hat{\mathcal{W}}_{2}}= & {\left[\begin{array}{llll}
6 & \mathfrak{p} 14 & 34 & \mathfrak{p} 82 \\
14 & 6 & 82 & 34 \\
34 & \mathfrak{p} 82 & 6 & \mathfrak{p} 14 \\
82 & 34 & 14 & 6
\end{array}\right], \quad \operatorname{det} \mathcal{B}_{\hat{\mathcal{W}}_{2}}=\mathfrak{p}^{2} 42614784-\mathfrak{p} 14623744+1254400, }
\end{aligned}
$$


and

$$
\hat{\mathcal{W}}_{2}^{\dagger_{1}}=\rho \hat{\mathcal{W}}_{2}=\left[\begin{array}{cccc}
1 & 0 & 0 & 0 \\
0 & -1 & 0 & 0 \\
0 & 0 & 1 & 0 \\
0 & 0 & 0 & -1
\end{array}\right]\left[\begin{array}{c}
6 \\
14 \\
34 \\
82
\end{array}\right]=\left[\begin{array}{llll}
6 & -14 & 34 & -82
\end{array}\right]^{T} .
$$

- $J=\varepsilon, \mathfrak{p}=0$ and $\overline{\mathcal{W}}_{2}(0,1 ; 3,2)=\overline{\mathcal{M}}_{2}$ Complex-dual Mersenne numbers:

$$
\begin{aligned}
\mathcal{X}_{\overline{\mathcal{W}}_{2}}= & {\left[\begin{array}{cc}
3+7 \varepsilon & -(15+31 \varepsilon) \\
15+31 \varepsilon & 3+7 \varepsilon
\end{array}\right], \quad \operatorname{det} \mathcal{X}_{\overline{\mathcal{W}}_{2}}=234+972 \varepsilon, } \\
\mathcal{D}_{\overline{\mathcal{W}}_{2}}= & {\left[\begin{array}{cccc}
3 & 0 & -15 & 0 \\
7 & 3 & -31 & -15 \\
15 & 0 & 3 & 0 \\
31 & 15 & 7 & 3
\end{array}\right], \quad \operatorname{det} \mathcal{B}_{\overline{\mathcal{W}}_{2}}=54756, }
\end{aligned}
$$

and

$$
\overline{\mathcal{W}}_{2}^{\dagger_{3}}=\tau \overline{\mathcal{W}}_{2}=\left[\begin{array}{cccc}
1 & 0 & 0 & 0 \\
0 & -1 & 0 & 0 \\
0 & 0 & -1 & 0 \\
0 & 0 & 0 & 1
\end{array}\right]\left[\begin{array}{c}
3 \\
7 \\
15 \\
31
\end{array}\right]=\left[\begin{array}{llll}
3 & -7 & -15 & 31
\end{array}\right]^{T} .
$$

\section{Conclusion}

In this paper, our own curiosity has been arisen by a desire to analyse the fundamentals for $\mathcal{D G C}, \mathcal{H G C}$, and $\mathcal{C G C}$ Horadam numbers. It should be noted that $\mathcal{D G C}$ Horadam numbers are a generalization of the $\mathcal{D G C}$ Fibonacci, Lucas, Pell, Pell-Lucas, Jacobsthal, Jacobsthal-Lucas and so on for $\mathfrak{p} \in \mathbb{R}$. Similar arguments are applied to $\mathcal{H G C}$ and $\mathcal{C G C}$ Horadam numbers and summarized briefly. The outstanding part of this paper is that, one can find well known identities and different matrix representations for all special types of Horadam numbers.

We hope readers will find interesting results if they examine $\mathcal{D G C}, \mathcal{H G C}$ and $\mathcal{C G C}$ Horadam numbers for special values of $\mathfrak{p} \in\{-1,0,1\}$, (see Table 4 ).

\begin{tabular}{|l||llrl|}
\hline Type & Horadam number & J & $\mathfrak{p}$ & Condition \\
\hline \hline Dual-complex & $W_{n}+W_{n+1} \mathbf{i}+W_{n+2} \varepsilon+W_{n+3} \mathbf{i} \varepsilon$ & $\mathbf{i}$ & -1 & \\
Hyper-dual & $W_{n}+W_{n+1} \varepsilon+W_{n+2} \varepsilon+W_{n+3} \varepsilon \varepsilon$ & $\varepsilon$ & 0 & $\varepsilon \neq 0, \boldsymbol{\varepsilon} \varepsilon \neq 0$ \\
Dual-hyperbolic & $W_{n}+W_{n+1} \mathbf{j}+W_{n+2} \varepsilon+W_{n+3} \mathbf{j} \varepsilon$ & $\mathbf{j}$ & 1 & $\mathbf{j} \neq \pm 1$ \\
Hyperbolic-complex & $W_{n}+W_{n+1} \mathbf{i}+W_{n+2} j+W_{n+3} \mathbf{i} j$ & $\mathbf{i}$ & -1 & \\
Hyperbolic-dual & $W_{n}+W_{n+1} \varepsilon+W_{n+2} j+W_{n+3} \varepsilon j$ & $\varepsilon$ & 0 & $\boldsymbol{\varepsilon} \neq 0$ \\
Bihyperbolic & $W_{n}+W_{n+1} \mathbf{j}+W_{n+2} j+W_{n+3} \mathbf{j} j$ & $\mathbf{j}$ & 1 & $\mathbf{j} \neq \pm 1, \mathbf{j} j \neq 1$ \\
Bicomplex & $W_{n}+W_{n+1} \mathbf{i}+W_{n+2} i+W_{n+3} \mathbf{i} i$ & $\mathbf{i}$ & -1 & $\mathbf{i} i \neq-1$ \\
Complex-dual & $W_{n}+W_{n+1} \varepsilon+W_{n+2} i+W_{n+3} \varepsilon i$ & $\varepsilon$ & 0 & $\boldsymbol{\varepsilon} \neq 0$ \\
Complex-hyperbolic & $W_{n}+W_{n+1} \mathbf{j}+W_{n+2} i+W_{n+3} \mathbf{j} i$ & $\mathbf{j}$ & 1 & $\mathbf{j} \neq \pm 1$ \\
\hline
\end{tabular}

Table 4. $\mathcal{D G C}, \mathcal{H G C}$ and $\mathcal{C G C}$ Horadam numbers for $\mathfrak{p} \in\{-1,0,1\}$ and $J \in\{\mathbf{i}, \boldsymbol{\varepsilon}, \mathbf{j}\}$ 


\section{References}

[1] Akar, M., Yüce, S., \& Şahin, S. (2018). On the dual hyperbolic numbers and the complex hyperbolic numbers. Journal of Computer Science \& Computational Mathematics, 8(1), $1-6$.

[2] Azak, A. Z. (2021). Some new identities with respect to Bihyperbolic Fibonacci and Lucas numbers. International Journal of Sciences: Basic and Applied Sciences, 60, 14-37.

[3] Belbachir, H., \& Belkhir, A. (2018). On some generalizations of Horadam's numbers. Filomat, 32(14), 5037-5052.

[4] Bród, D., Szynal-Liana, A., \& Włoch, I. (2021). On some combinatorial properties of bihyperbolic numbers of the Fibonacci type. Mathematical Methods in the Applied Sciences, 44, 4607-4615.

[5] Catoni, F., Boccaletti, D., Cannata, R., Catoni, V., Nichelatti, E., \& Zampetti, P. (2008). The Mathematics of Minkowski Space-Time with an Introduction to Commutative Hypercomplex Numbers. Birkhauser Verlag, Basel, Boston, Berlin.

[6] Cheng, H. H., \& Thompson, S. (1999). Singularity analysis of spatial mechanisms using dual polynomials and complex dual numbers. Journal of Mechanical Design, 121(2), 200-205.

[7] Cihan, A., Azak, A. Z., Güngör, M. A., \& Tosun, M. (2019). A study on dual hyperbolic Fibonacci and Lucas numbers. Analele Universitatii “Ovidius” Constanta-Seria Matematica, 27(1), 35-48.

[8] Cockle, J. (1849). On a new imaginary in algebra. The London, Edinburgh, and Dublin Philosophical Magazine and Journal of Science, 34(226), 37-47.

[9] Cohen, A., \& Shoham, M. (2018). Principle of transference-An extension to hyper-dual numbers. Mechanism and Machine Theory, 125, 101-110.

[10] Fike, J. A., \& Alonso, J. J. (2012). Automatic differentiation through the use of hyper-dual numbers for second derivatives. In: Forth S., Hovland P., Phipps E., Utke J., Walther A. (eds) Recent Advances in Algorithmic Differentiation. Lecture Notes in Computational Science and Engineering, Springer, Berlin, Heidelberg, 87, 163-173.

[11] Güngör, M. A., \& Azak, A. Z. (2017). Investigation of dual-complex Fibonacci, dual-complex Lucas numbers and their properties. Advances in Applied Clifford Algebras, 27(4), 3083-3096.

[12] Gürses, N., Şentürk, G. Y., \& Yüce, S. (2021). A study on dual-generalized complex and hyperbolic-generalized complex numbers. Gazi University Journal of Science, 34(1), 180-194. 
[13] Gürses, N., Şentürk, G. Y., \& Yüce, S. (2021). A comprehensive survey of dual-generalized complex Fibonacci and Lucas numbers. Sigma Journal of Engineering and Natural Sciences, (in press).

[14] Halıc1, S. (2019). On Bicomplex Fibonacci Numbers and Their Generalization, Models and Theories in Social Systems. Studies in Systems, Decision and Control, 179, Springer, Cham.

[15] Harkin, A. A., \& Harkin, J. B. (2004). Geometry of generalized complex numbers. Mathematics magazine, 77(2), 118-129.

[16] Haukkanen, P. (2002). A note on Horadam's sequence. The Fibonacci Quaterly, 40(4), $358-361$.

[17] Horadam, A. F. (1961). A generalized Fibonacci sequence. The American Mathematical Monthly, 68(5), 455-459.

[18] Horadam, A. F. (1965). Generating functions for powers of a certain generalised sequence of numbers. Duke Mathematical Journal, 32(3), 437-446.

[19] Horadam, A. F. (1965). Basic properties of a certain generalized sequence of numbers. The Fibonacci Quarterly, 3, 161-176.

[20] Horadam, A. F. (1967). Special properties of the sequence $W_{n}(a, b ; p, q)$. The Fibonacci Quarterly, 5, 424-434.

[21] Kantor, I., \& Solodovnikov, A. (1989). Hypercomplex numbers, Springer-Verlag, New York.

[22] Kılıç, E., \& Tan, E. (2015). On binomial sums for the general second order linear recurrence. Integers, 10(6), 801-806.

[23] Kılıç, E., \& Tan, E. (2009). More general identities involving the terms of $\left\{W_{n}(a, b ; p, q)\right\}$. Ars Combinatoria, 93, 459-461.

[24] Köme, S., Köme, C., \& Yazlik, Y. (2021). Dual-complex generalized k-Horadam numbers. Communications Faculty of Sciences University of Ankara Series A1 Mathematics and Statistics, 70(1), 117-129.

[25] Majernik, V. (1996). Multicomponent number systems. Acta Physica. Polonica A 90(3), 491-498.

[26] Messelmi, F. (2015). Dual-complex numbers and their holomorphic functions. Preprint. HAL ID: hal-01114178.

[27] Mező, I. (2009) Several generating functions for second-order recurrence sequences, Journal of Integer Sequences, 12, Article 09.3.7.

[28] Ömür, N., \& Koparal, S. (2020). On hyper-dual generalized Fibonacci numbers. Notes on Number Theory and Discrete Mathematics, 26(1), 191-198. 
[29] Pennestrì, E., \& Stefanelli, R. (2007). Linear algebra and numerical algorithms using dual numbers. Multibody System Dynamics, 18(3), 323-344.

[30] Rayaguru, S. G., Savin, D., \& Panda, G. K. (2019). On some Horadam symbol elements. Notes on Number Theory and Discrete Mathematics, 25(2), 91-112.

[31] Study, E. (1903). Geometrie der dynamen: Die zusammensetzung von kräften und verwandte gegenstände der geometrie bearb. Leipzig, B.G. Teubner. 1903.

[32] Sobczyk, G. (1995). The hyperbolic number plane. The College Mathematics Journal, 26(4), $268-280$.

[33] Tan, E., Ait-Amrane, N. R., \& Gök, I. (2021). Hyper-Dual Horadam quaternions. Preprints, 2021030435.

[34] Taşkara, N., Uslu, K., Yazlık, Y., \& Yılmaz, N. (2011). The construction of Horadam numbers in terms of the determinant of tridiagonal matrices. AIP Conference Proceedings, 1389(1), 367-370.

[35] Yaglom, I. M. (1968). Complex numbers in geometry. Academic Press, New York.

[36] Yaglom, I. M. (1979). A simple non-Euclidean geometry and its physical basis. SpringerVerlag, New York.

[37] Yazlık, Y., \& Taskara, N. (2012). A note on generalized $k$-Horadam sequence. Computers \& Mathematics with Applications, 63(1), 36-41. 